\title{
ABILITY OF ENERGY HARVESTING MR DAMPER TO ACT AS A VELOCITY SENSOR IN VIBRATION CONTROL SYSTEMS
}

\author{
Maciej ROSÓŁ, "Bogdan SAPIŃSKI ${ }^{*}$ \\ ${ }^{*}$ Faculty of Electrical Engineering Automatics Computer Science and Biomedical Engineering, Department of Automatic Control and Robotics, \\ AGH University of Science and Technology, Al. Mickiewicza 30, 30-059 Kraków, Poland \\ "Faculty of Mechanical Engineering and Robotics, Department of Process Control, AGH University of Science and Technology, \\ Al. Mickiewicza 30, 30-059 Kraków, Poland \\ mr@agh.edu.pl, deep@agh.edu.pl
}

received 12 April 2019, revised 26 June 2019, accepted 28 June 2019

\begin{abstract}
The study investigates the self-sensing ability in an energy harvesting magnetorheological damper (EHMRD). The device consists of a conventional linear MR damper and an electromagnetic harvester. The objective of the work is to demonstrate that the EHMRD with specific self-powered feature can also serve as a velocity sensor. Main components of the device and design structure are summarized and its operation principle is highlighted. The diagram of the experimental set-up incorporating the measurement and processing unit is provided, the experimental procedure is outlined and data processing is discussed. The self-sensing function is proposed whereby the relative velocity of the EHMRD can be reconstructed from the electromotive force (emf) induced in the harvester coil. To demonstrate the adequacy of the self-sensing action (i.e., the induced emf should agree well with the relative velocity), the proposed self-sensing function is implemented and tested in the embedded system that will be a target control platform. Finally, the test results of the system utilizing a switching control algorithm are provided to demonstrate the potentials of the EHMRD acting as a velocity sensor and to confirm its applicability in semi-active vibration control systems.
\end{abstract}

Key words: MR damper, energy harvester, self-sensing function, electromotive force, embedded system, algorithm

\section{INTRODUCTION}

It is a well-established fact that vibrating objects such as machines, equipment, vehicles, engineering structures and so on generate mechanical energy that might be used as an energy source. This energy, otherwise wasted, could be converted into usable electrical energy to power sensors or/and actuators, thus eliminating the need of external energy power sources, making the systems self-powered. Inspired by this concept, researches have already achieved the most promising results in the energy harvesting area.

Most studies on EHMRDs have focused on their self-powered ability whilst less attention has been given to their self-sensing feature. According to the authors, this aspect seems of great importance too, meriting full attention and extensive research efforts. The present study investigates this specific feature in the engineered MR damper integrated with an electromagnetic energy harvester (Sapiński, 2014). Formerly, this damper was tested in a semi-active vibration control system employed in a 1 DOF mechanical structure configuration but research efforts at that time focused mainly on the self-powering feature of the device (Sapinski and Rosół, 2016).

The last decade has witnessed a major interest in EHMRDs. A growing number of research reports have been published, summarizing the developments and achievements in this area. It is worthwhile to mention that several solutions have already been patented. Recent advancements in the area of EHMRDs have been summarized in the work by Ahamed et al. (2016). This comprehensive review shows that the concept of MR dampers with power generation still remains a challenging problem. Moreover, extensive research efforts have been made to implement EHMRDs in automobiles, railway vehicles and so on, and to evaluate their performance. A thorough review of recent research reports on the subject is presented below.

Choi and Werely (2009) investigated the feasibility and efficiency of a self-powered MR damper using a spring-mass electromagnetic energy harvester. Zhu et al. (2012) investigated the feasibility of linear motion electromagnetic devices for civil infrastructure to be used for vibration damping and energy harvesting. Jung et al. (2010a) demonstrated that an electromagnetic energy harvester incorporated in the MR damper-based system may act as a velocity-sign sensor. Also, Jung et al. (2010b) showed that such a harvester could act as a relative velocity sensor in typical control strategies for MR damper-based systems. Wang et al. (2010) proposed an integrated relative displacement self-sensing MR damper to realize the integrated relative displacement sensing and controllable damping. Wang and Bai (2013) developed this idea and fabricated and tested such an MR damper prototype. Peng et al. (2011) formulated the concept and proposed the optimization of the self-sensing MR damper that superposes the information of the damper's motion to the emf signal, which can be further modulated via the feedback strategy. Chen and Liao (2012) investigated a newly developed MR damper prototype with self-powered and self-sensing abilities. Liao and Chen (2010) accordingly filed the patent application for the conceptual design of the device. Zhu et al. (2012) studied the self-powered and sensor-based MR damper systems, which could be particularly useful in large-scale civil structures where the power supply is impractical. Li et al. (2013a) presented an innovative concept of a 
so-called mechanical motion rectifier, which converts bidirectional motion into unidirectional motion. Li et al. (2013b) developed a retrofittable design of a shock absorber based on permanent magnets and rack-pinion mechanism. Ni et al. (2015) developed and identified inverse dynamics of a self-sensing MR damper that may be applicable for structural control applications. Chen et al. (2015) presented a self-sensing MR damper with an embedded piezoelectric force sensor and demonstrated its reliable force sensing and controllable damping capabilities by the use of NARX modelling and neural network techniques. Hu et al. (2015) developed a novel MR damper with a self-induced ability integrated with a linear variable differential sensor based on the electromagnetic induction mechanism and evaluated its static and dynamic behaviour through experimental testing. Also, Hu et al. (2015) proposed a self-sensing MR damper with an electromagnetic induction mechanism, which has both good self-sensing ability and controllable damping capability. Xinchun et al. (2015) proposed an MR damper with a self-powered ability equipped with vibration energy harvesting mechanism based on ball-screw and a rotary permanent magnet dc generator, and indicated the feasibility of this configuration. Xinchun et al. (2017) developed the MR damper with velocity self-sensing mechanism based on the optical tracking technology and numerical circuit technology, and demonstrated its high accuracy monitoring capability and sufficient ability to control the MR damper. Ahamed et al. (2016) proposed an MR damper with the energy harvesting component, and through the tests, revealed the maximum induced output voltage.

The objective of the present study is to demonstrate that the engineered EHMRD that exhibits self-powered ability can also act as a velocity sensor in vibration control systems. The work is organized in the following manner. Section 2 outlines the structure of the EHMRD. Section 3 provides the harvester configuration, basic geometry and electrical parameters, and highlights its operating principles. Section 4 summarizes the experimental set-up description, the measurement and processing unit, the experi- mental and data analysis procedures. In Section 5 , the main focus is on the self-sensing function, its identification and verification. This function has been implemented in the STM32F407 processor embedded system and the application with switching control algorithm is shown to confirm the harvester performance as a velocity sensor. Final conclusions are provided in Section 6.

\section{STRUCTURE OF THE EHMRD}

The cut-off view of the EHMRD is shown in Fig. 1. The device is symmetrical and incorporates two major components: the harvester and the MR damper. A piston rod (1) made of ferromagnetic steel moves inside a brass lid (2). Rubber seals (3) prevent MR fluid leaking. The rod is bolted to the piston (4). In the piston, there is the MR damper control coil (5) power-supplied via a wire (6) in the rod. The piston moves inside the damper housing (7) made of ferromagnetic steel and connected to the harvester housing (8) made of aluminium, via a brass connector (9). The piston is connected to the harvester via a rod (10) made of non-magnetic steel. On the rod in the harvester are three arrays of ring-shaped neodymium magnets, magnetized axially (11), separated from one another by spacers (12) made of ferromagnetic steel. The diameter of the magnets is identical to that of the spacers. Magnets and the rod move inside the immobile housing (13) made of ferromagnetic steel. The harvester's coil (14), wound on the carcass and placed inside the housing, has two-sections with copper foil. The sections are connected such that induced emfs should sum up. The middle point along the height of the sections coincides with the point half-way between the magnet arrays. The aluminium lid (15) locks the harvester coil housing in place. Linear guiding of the damper rod and harvester is affected through the use of slip sleeves (16). A holder (17) is bolted to the lid in the damper, enabling its assembly in various configurations.

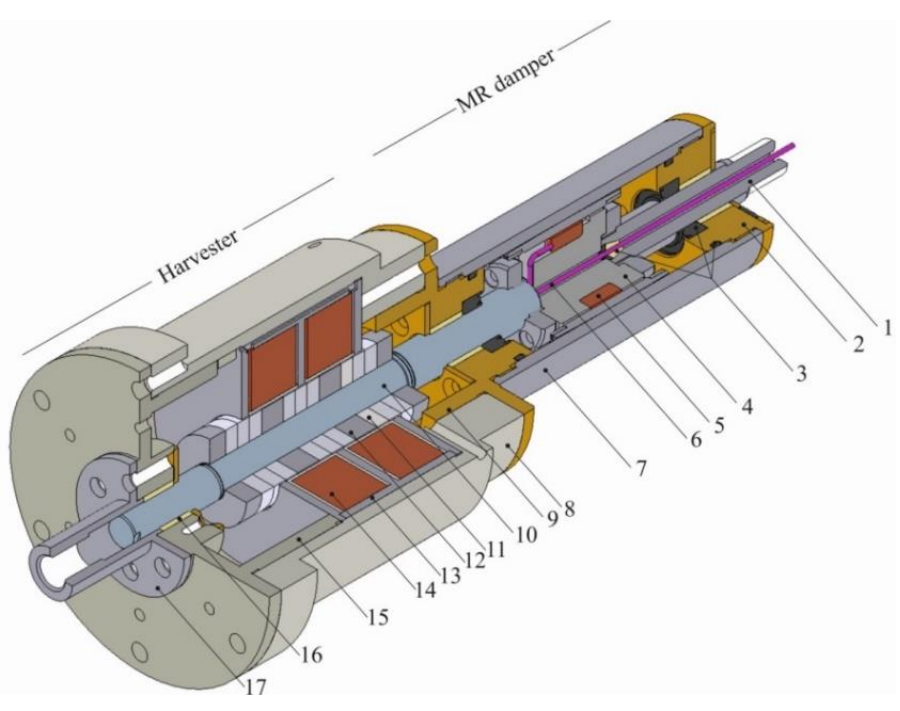

Fig. 1. EHMRD; cut-off view

\section{CONFIGURATION AND OPERATING PRINCIPLE OF THE HARVESTER}

This configuration of the harvester has evolved as the results of extensive research efforts collated in Sapiński (2008; 2010, 2013). These studies summarized the numerical calculations, design considerations, fabrication and experimental testing procedures, and engineering of the electromagnetic harvester for a linear MR damper. The present harvester configuration is shown in Fig. 2a. Dimensions of its main components are as follows: 
magnets $\left(h_{m}=5 \mathrm{~mm}, d_{m i}=20 \mathrm{~mm}, d_{m e}=80 \mathrm{~mm}\right)$, coil $\left(H_{c}=54\right.$ $\left.\mathrm{mm}, d_{c i}=86 \mathrm{~mm}, d_{c e}=127.6 \mathrm{~mm}\right)$, spacers $\left(H_{g}=20 \mathrm{~mm}\right)$, carcass $\left(H_{c a}=58 \mathrm{~mm}, g_{c a}=2 \mathrm{~mm}\right)$, coil housing $\left(H_{h}=86 \mathrm{~mm}, w_{h}=3 \mathrm{~mm}\right)$. The copper foil used in the harvester coil is $50 \mathrm{~mm}$ in width and $0.05 \mathrm{~mm}$ in thickness, with one-sided insulation $54 \mathrm{~mm}$ wide and $0.03 \mathrm{~mm}$ thick. The winding sections have identical height and the number of turns is 273 . The height of an air slit between the carcass and the system of magnets is $1 \mathrm{~mm}$.
Fig. $2 b$ illustrates the magnetic flux distribution calculated with respect to the centre of the harvester coil winding sections (Coil $\mathrm{H} 1$ and Coil $\mathrm{H} 2$ ). It is apparent that the harvester coil's housing and a portion of the magnets is permeated by the magnetic flux linkage encompassing the coil turns. The magnet systems assembly and coil assembly of the harvester are shown in Figs. 2c, d.

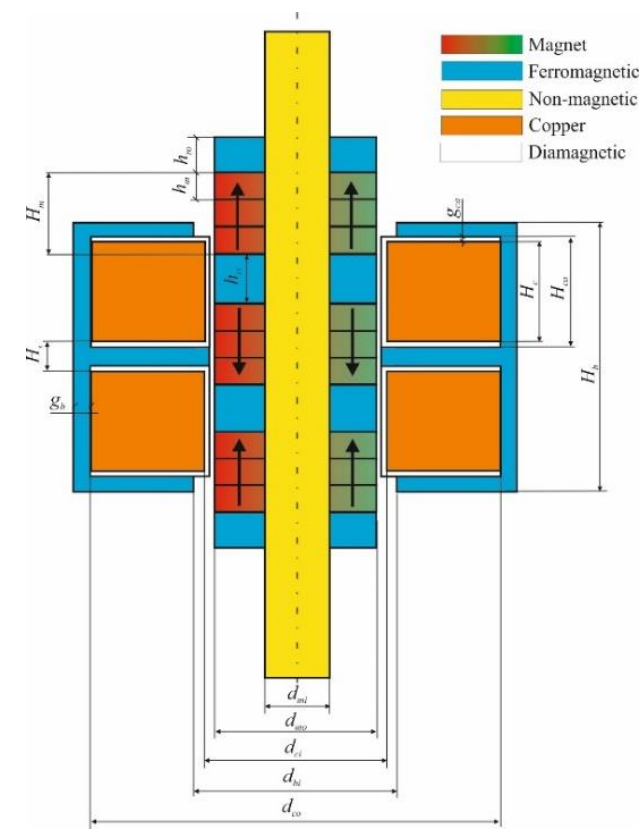

(a)

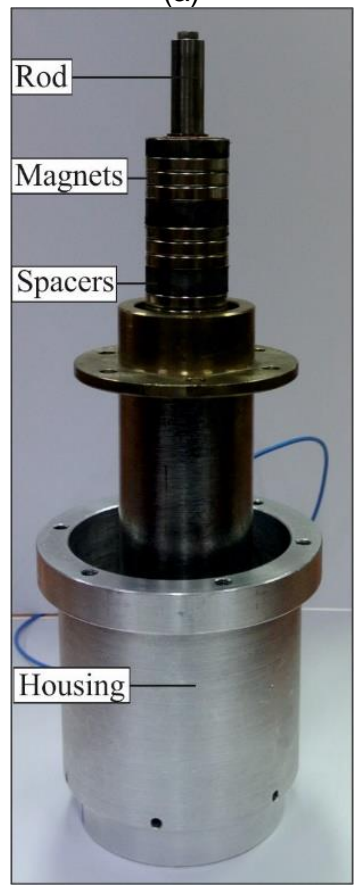

(c)

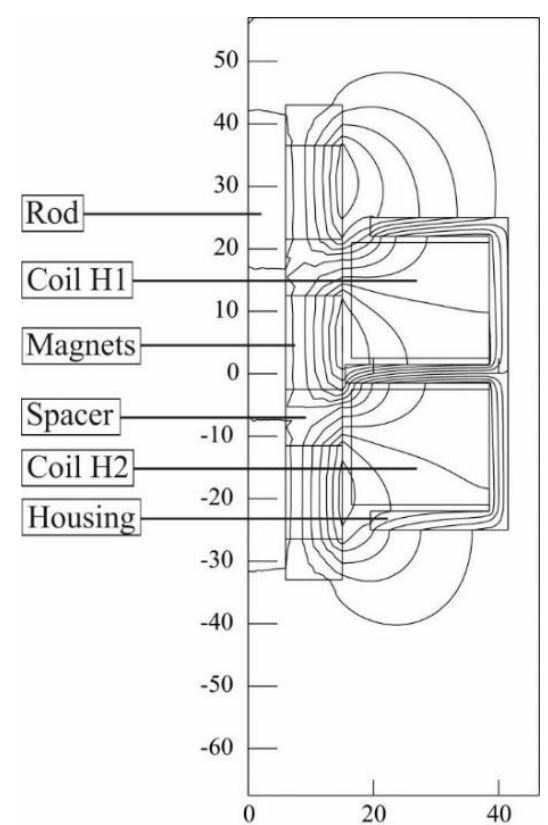

(b)

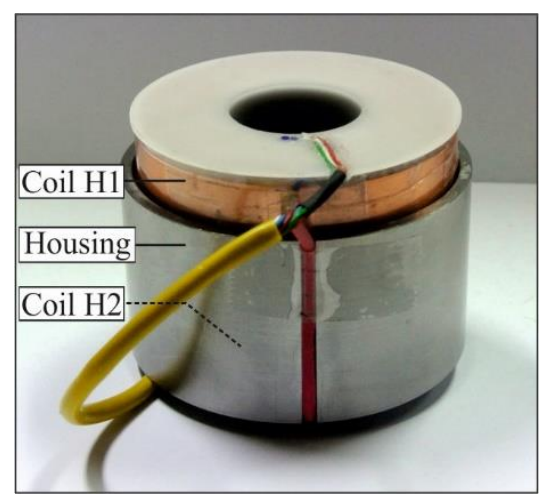

(d)

Fig. 2. Harvester: (a) schematic diagram, (b) magnetic flux density map, (c) magnet systems assembly, (d) coil assembly

The operating principle of the harvester unit relies on Faraday's law, which states that relative motion of stationary magnets with respect to the coil will induce an emf. This emf is proportional to the velocity across the device, and hence, it may serve as a velocity sensor. The concept of using the harvesting component to reconstruct the velocity signal and to supply the MR damper is illustrated in an equivalent circuit in Fig. 3. For simplicity, let us assume that all circuit components are linear. When the switch contact is in the 'close' position, Coil H1 (resistance $R_{1}$ and inductance $L_{1}$ in series) acts as a measuring coil since it will have 
the induced emf $e$. When the switch contact is in the 'open' position, Coil $\mathrm{H} 2$ (resistance $R_{2}$ and inductance $L_{2}$ in series) acts as the coil supplying the MR damper (resistance $R_{d}$ and inductance $L_{d}$ in series). Depending on the switch position, the EHMRD operates either under idle run (as a sensor) or under the load (as an energy source). One has to bear in mind that the core loss effect in the device has been neglected because of low-frequency excitations during the tests. In order to account for core loss effects, it is required that an equivalent resistance in parallel with inductance $L_{d}$ should be incorporated in the circuit in Fig. 3. Resistance of the harvester coil (Coil $\mathrm{H} 1$ and Coil $\mathrm{H} 2$ ) becomes $R_{1}+R_{2}=2.45$ $\Omega$ and inductance $L_{1}+L_{2}=141 \mathrm{mH}$. Resistance of the MR damper coil is $R_{d}=3.6 \Omega$ and inductance $L_{d}=71 \mathrm{mH}$.

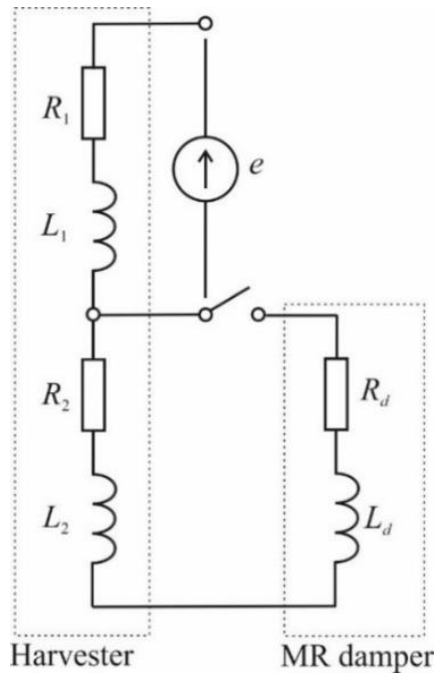

Fig. 3. Equivalent circuit of the EHMRD under idle run and under the load

\section{EXPERIMENTS AND DATA ANALYSIS}

To obtain the data requisite for the reconstruction of velocity across the EHMRD, measurements were taken of emf induced in the harvester coil in the experimental set-up incorporating a testing machine MTS 810 controlled via a FlexTest SE controller (MTS, 2006). The diagram of the experimental set-up is shown in Fig. 4. The measurement unit based on high-precision operational amplifiers was used for gain regulation and to offset the measured analogue quantities. The processing unit, based on the STM32F407 processor, has all the requisite peripheral systems ensuring the sufficient computational capacity of CPU and FPU units (STMicroelectronics, 2017). Embedded system was tested using the following peripheral blocks integrated in the structure: analogue-digital converter (ADC), timers/counters units capable of interrupts and PWM signal generation, and a serial port UART. Two channels of a 12-bit A/D converter are responsible for velocity and emf measurements, conditioned by the measurement unit. The measurement unit converts the measured voltages into voltages from the range $(0,+3.3) \mathrm{V}$, acceptable by STM32F407 processor. Thus, the processed data are duly transmitted (via an UART port), monitored and uploaded to a PC. Recorded data are sampled with the frequency of $1 \mathrm{kHz}$ (periodical interrupt-driven function). Four additional digital outputs pin of STM32F407 processor are used to measure the sample time period $\left(T_{0}\right)$, time duration of velocity reconstruction from emf $\left(T_{A}\right)$, time duration of control algorithm $\left(T_{C}\right)$ and time of data transmission $\left(T_{S}\right)$.

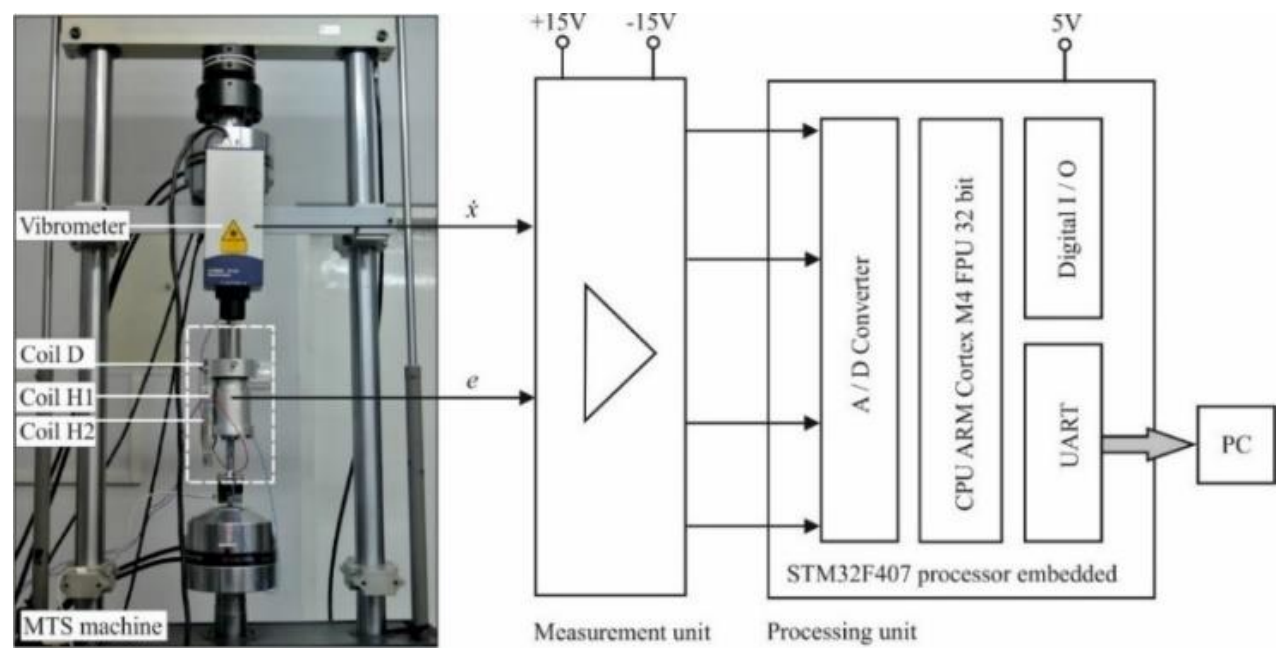

Fig. 4. Diagram of the experimental set-up

Measurement data were registered in the two modes of the EHMRD's operation: under idle run and under the load, the applied inputs were sine and triangular displacements of the piston rod with the amplitude $5 \mathrm{~mm}$ and frequency varied from 1 to $7 \mathrm{~Hz}$ with $1 \mathrm{~Hz}$ increments. These levels of input amplitude and frequency were designed in the context of potential implementation of the EHMRD in a semi-active vibration reduction system. Velocity $\dot{x}$ was measured with a laser vibrometer (Polytec, 2005), with resolution $0.1 \mu \mathrm{m} / \mathrm{s}$ (the level for $1 \mathrm{~Hz}$ ) for the ADC voltage range $\pm 10 \mathrm{~V}$. Measurement data were recorded, analysed and processed using a PC computer supported by MATLAB/Simulink.

To reconstruct the velocity across the EHMRD, the authors propose the self-sensing function that is determined in a multistage procedure. Stage 1 consisted of the initialization of the peripheral systems and parameters of the processing unit. In Stage 2, the type and parameters of the input excitations (piston 
rod displacement $x$ ) were selected, alongside the operating mode of the device (under idle run or under the load). In Stage 3 , the velocity $\dot{x}$ and emf data registered under idle run $(e)$ and under the load $\left(e_{L}\right)$ were uploaded to a PC. Stage 4 involved the analysis of measurement data supported by MATLAB; moreover, the actual form and parameters of the self-sensing function were determined accordingly. In Stage 5, the self-sensing function was verified using a different set of data.

In Stage 4, the measurement data were analysed and processed in the time and frequency domain. The time-domain analysis involved the comparison of time histories of velocity $\dot{x}$ and emf $e$, in the case of the frequency domain analysis, the Digital Fourier Transform (DFT) values of respective quantities were compared for the assumed inputs excitations $x$. DFT modulus was calculated using the fft.m of MATLAB function based on the Cooley-Tukey algorithm (Frigo and Johnson, 2005). The number equal to the nearest power of 2 greater than the length of the registered data was used as the FFT points number. The missing data of an input vector are padded with trailing zeros to the determined FFT length.

Figs. 5 a, b show time histories of velocity $\dot{x}$, emf $e$ (under idle run) and $e_{L}$ (under the load) applied to the Coil $\mathrm{H} 1$, at frequency 1 $\mathrm{Hz}$ and $7 \mathrm{~Hz}$. These quantities are found to be in phase and they reach the zero value at identical time instants. Differences between time histories of $e$ and $e_{L}$ appear to be minor. The maximal relative difference approaches 0.1052 at $1 \mathrm{~Hz}$ and 0.1748 at $7 \mathrm{~Hz}$. The integral of the squared error per period is 2.2293 at $1 \mathrm{~Hz}$ and 0.9561 at $7 \mathrm{~Hz}$. Similar differences and error values are registered at other frequencies.

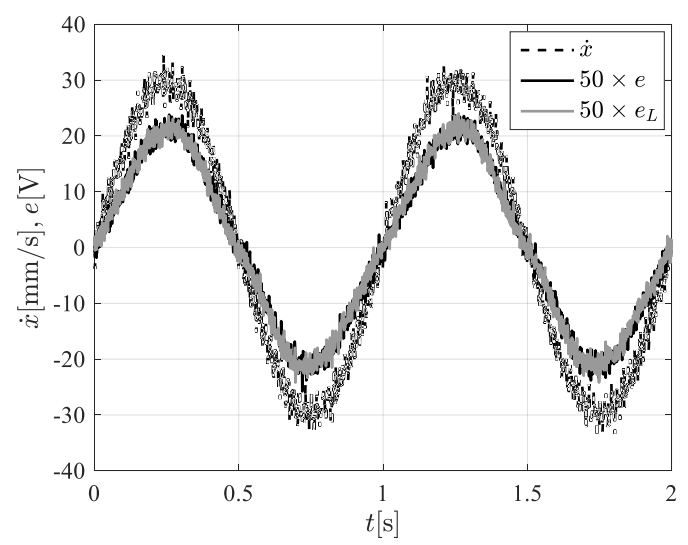

(a) sine input, $f=1 \mathrm{~Hz}$

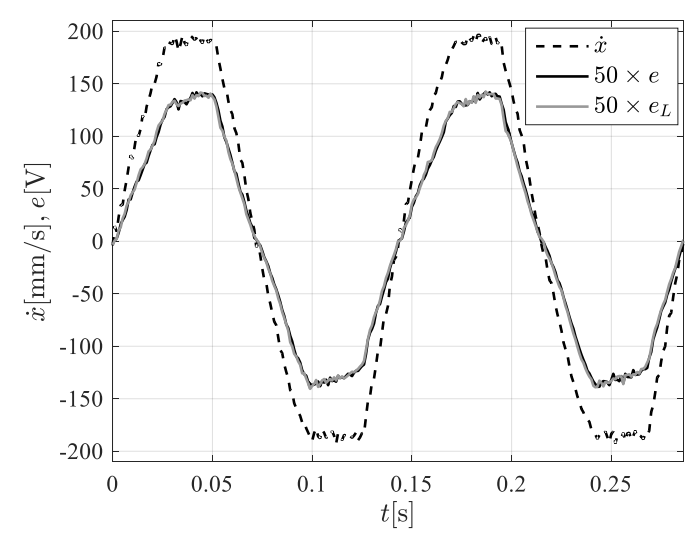

(b) sine input, $f=7 \mathrm{~Hz}$

Fig. 5. Time history of velocity and emf; sine input
Figs. 6 a, b plot the DFT modulus of $|\dot{X}(f)|$ and emf under idle run $|E(f)|$ and under the load $\left|E_{L}(f)\right|$ applied to the coil $\mathrm{A}$, at frequency 1 and $7 \mathrm{~Hz}$. Apparently, the differences between DFT modulus of $|E(f)|$ and $\left|E_{L}(f)\right|$ are rather small. The maximal relative difference is 0.32 at $1 \mathrm{~Hz}$ and 0.2 at $7 \mathrm{~Hz}$. The integral of the squared error calculated at frequency range $(1,10) \mathrm{Hz}$ equals 0.0018 at $1 \mathrm{~Hz}$ and 0.011 at $7 \mathrm{~Hz}$. Similar results were obtained at other frequencies.

It is reasonable to suppose, therefore, that the time history of rod velocity $x^{*}$ could be reconstructed from emf readouts $e$.

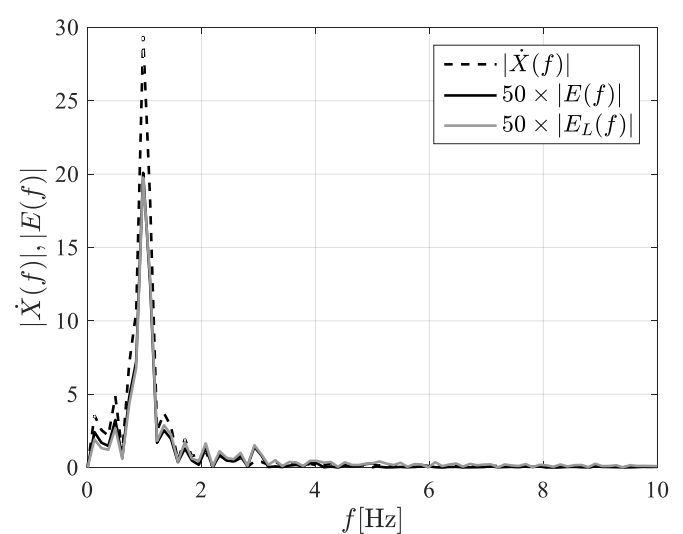

(a) $f=1 \mathrm{~Hz}$

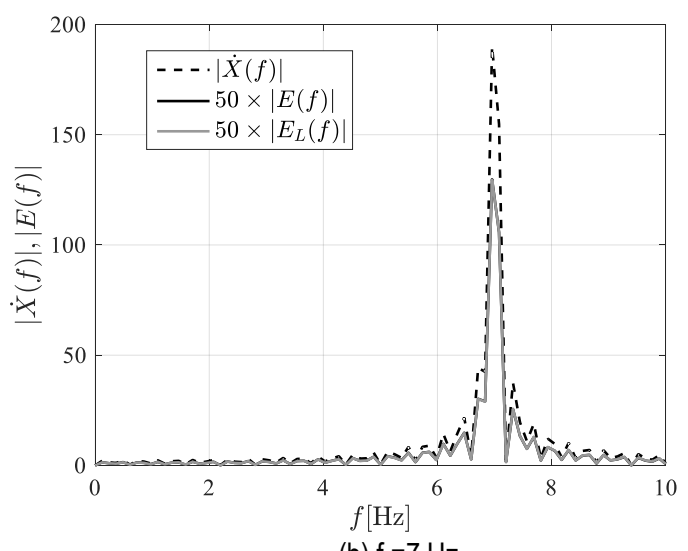

(b) $f=7 \mathrm{~Hz}$

Fig. 6. DFT values of velocity and emf; sine input

\section{VELOCITY RECONSTRUCTION}

\subsection{Identification of self-sensing function}

Following the discussion, the self-sensing function $\dot{x}=f(e)$ is proposed, defined by the first-order polynomial. When analysing the problem in time domain, the first harmonic amplitude of the velocity $\dot{X}_{1}$ and emf $\dot{E}_{1}$ calculated from the time series at each frequency in the range $(1,7) \mathrm{Hz}$ yields the formula:

$\dot{X}_{1}=a E_{1}+b$

In frequency domain, the first harmonic modulus of the velocity $\left|\dot{X}_{1}(f)\right|$ and emf $\left|E_{1}(f)\right|$, determined from DFT series at each frequency in the range $(1,7) \mathrm{Hz}$, yields the formula:

$\left|\dot{X}_{1}(f)\right|=a\left|E_{1}(f)\right|+b$

The values of parameters $a$ and $b$ calculated by the least square 
method (polyfit function available in MATLAB) are summarized in Table 1. Parameters $a$ and $b$ were obtained in the time domain and in the frequency domain for the sets of data registered under idle run $\left(S_{t}^{1}, S_{f}^{1}\right)$, under the load $\left(S_{t}^{2}, S_{f}^{2}\right)$ and jointly for the two modes $\left(S_{t}^{3}, S_{f}^{3}\right)$. The goodness of fit of $\dot{X}_{1}$ and $\left|\dot{X}_{1}(f)\right|$ to the predicted values derived from (1) and (2) was evaluated recalling the Pearson's linear correlation coefficient $r$. Fig. 7, 8 show the relationship (1) and (2) obtained for $S_{t}^{1}$ and $S_{f}^{1}$ data set.

Tab. 1. Calculated parameters $a, b$ and correlation coefficient $r$

\begin{tabular}{|c|c|c|c|c|}
\hline Domain & Data set & $\boldsymbol{a}$ & $\boldsymbol{b}$ & $\boldsymbol{r}$ \\
\hline \multirow{3}{*}{ Time } & $S_{t}^{1}$ & 73.24 & 0.011 & 0.9999993 \\
\cline { 2 - 5 } & $S_{t}^{2}$ & 73.35 & 0.058 & 0.99999899 \\
\cline { 2 - 5 } & $S_{t}^{3}$ & 73.31 & 0.035 & 0.9999981 \\
\hline \multirow{3}{*}{ Frequency } & $S_{f}^{1}$ & 73.25 & -0.0054 & 0.9999992 \\
\cline { 2 - 5 } & $S_{f}^{2}$ & 73.34 & 0.044 & 0.9999993 \\
\cline { 2 - 5 } & $S_{f}^{3}$ & 73.29 & 0.025 & 0.9999461 \\
\hline
\end{tabular}

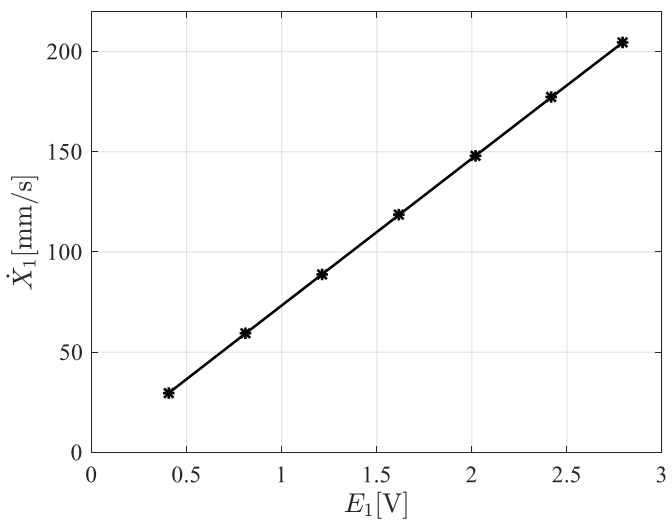

Fig. 7. First harmonic amplitude of velocity vs. first harmonic amplitude of emf

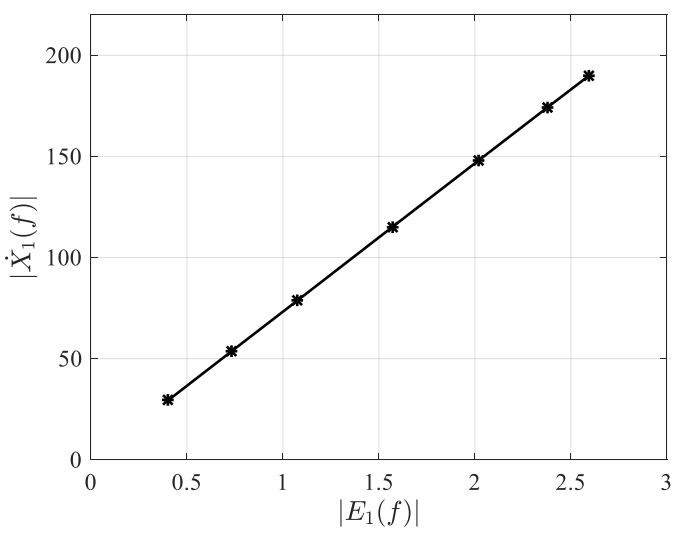

Fig. 8. Modulus of first harmonic of velocity vs. modulus first harmonic of emf

Actual values of parameters $a$ and $b$ in Table 1 were selected based on the computed values of MSE (Mean-Squared Error) between the measured velocity $\dot{x}$ and reconstructed velocity $\dot{x}_{e}$ defined as follows:

$M S E=\frac{1}{M} \sum_{i=1}^{M}\left(\dot{x}_{i}-\dot{x}_{e i}\right)^{2}$ where $M$ is the number of samples per a single period of input displacement $x(t)$.

Each pair of values of parameters $a$ and $b$ (Table 1$)$ was recalled to compute the velocity $\dot{x}_{e}$ reconstructed from $e$ for two data sets registered under idle run and under the load for each frequency in the range $(1,7) \mathrm{Hz}$.

Fig. 9 gives the medium MSE error computed for each data set, in accordance with the formula:

$S_{D}^{m, n}=\frac{1}{K} \sum_{i=1}^{K} \operatorname{MSE}(i)$

where: $K=7$ is the number of input frequencies; $D=t$ in time domain or $D=f$ in frequency domain; $m, n=1,2,3$ are data sets used to obtain the parameters $a$ and $b(m)$ and to reconstruct velocity from emf $(n)$.

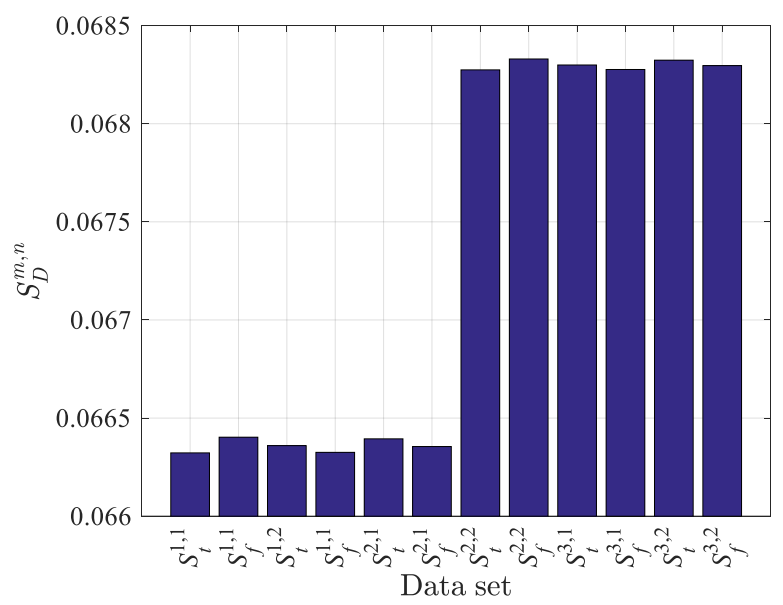

Fig. 9. Medium value of MSE error calculated for each data set

It appears that the smallest error value $S_{D}^{m, n}$ is obtained for a and $b$ values derived for the data set $S_{t}^{1,1}$, that is why these values of $a$ and $b$ were recalled in further considerations. Fig. 10a, $10 \mathrm{~b}$ compare the plots of measured velocity $\dot{x}$ with that reconstructed from formula (1) $\dot{x}_{e}$ for sine excitation inputs and at frequency $1 \mathrm{~Hz}$ and $7 \mathrm{~Hz}$. Recalling the switching algorithms widely used in semi-active vibration control systems, such as sky-hook and ground-hook algorithms (Karnopp et al., 1974) expressed by formula (5) and (6), it is clearly apparent that in order to calculate the control $u_{\text {sky }}$ or $u_{\text {grd }}$, the velocity $\dot{x}_{e}$ should be determined with sufficient precision.

$u_{s k y}=\left\{\begin{array}{c}u_{s} ; \dot{x}_{s} \cdot \dot{x}_{e} \geq 0 \\ 0 ; \dot{x}_{s} \cdot \dot{x}_{e}<0\end{array}\right.$

$u_{\text {grd }}=\left\{\begin{array}{c}u_{g} ;-\dot{x}_{u} \cdot \dot{x}_{e} \geq 0 \\ 0 ;-\dot{x}_{u} \cdot \dot{x}_{e}<0\end{array}\right.$

where $\dot{x}_{s}$ is velocity of a sprung mass and $\dot{x}_{u}$ is velocity of an unsprung mass.

In the context of these algorithms, of particular importance are the points where the velocity sign should change. The maximum time between the instants when the signs of velocity $\dot{x}$ and $\dot{x}_{e}$ should change is found to be $600 \mu \mathrm{s}$, determined from the respective time histories. This value is almost equal to the sampling period $1 \mathrm{~ms}$. 


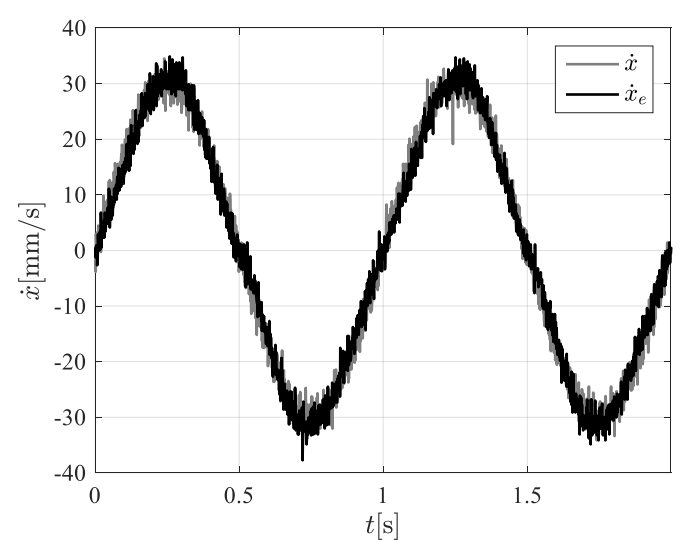

(a) $f=1 \mathrm{~Hz}$

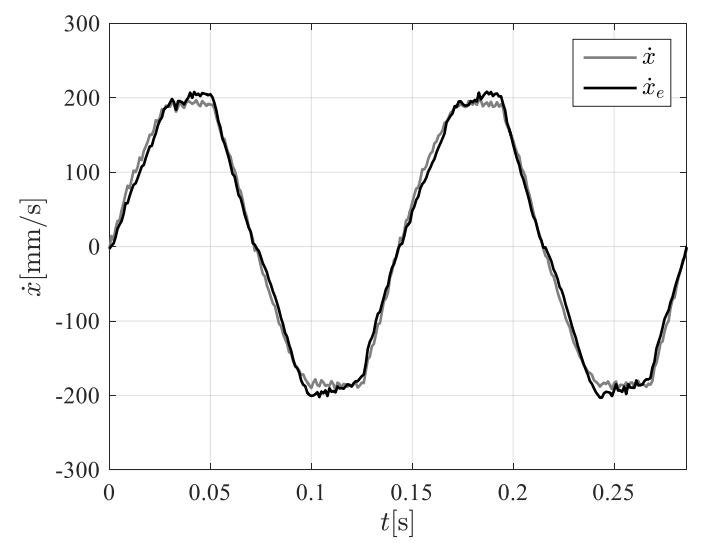

(b) $f=7 \mathrm{~Hz}$

Fig. 10. Time history of velocity measured and reconstructed from emf; sine input

\subsection{Verification of self-sensing function}

In order to verify the introduced self-sensing function, the triangle displacement input was applied. Figs. 11, 12 plot velocity $\dot{x}$ readout from vibrometer and the reconstructed velocity obtained under idle run $\dot{x}_{e}$ and under the load $\dot{x}_{e L}$. It appears that the time instants when the velocity sign is changed are determined with the level of accuracy $600 \mu \mathrm{s}$. Most significant differences are registered for constant values of velocity $\dot{x}_{e}$ and $\dot{x}_{e L}$, which is associated with the steady-state emf value having approached emf $(e)$.

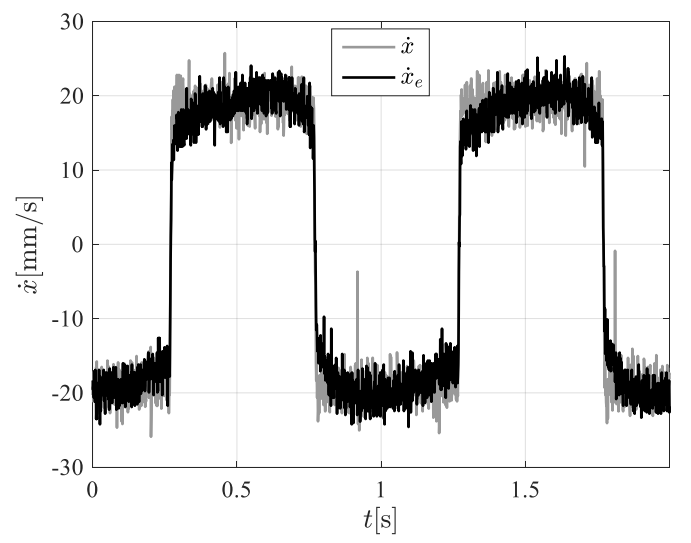

(a) under idle run

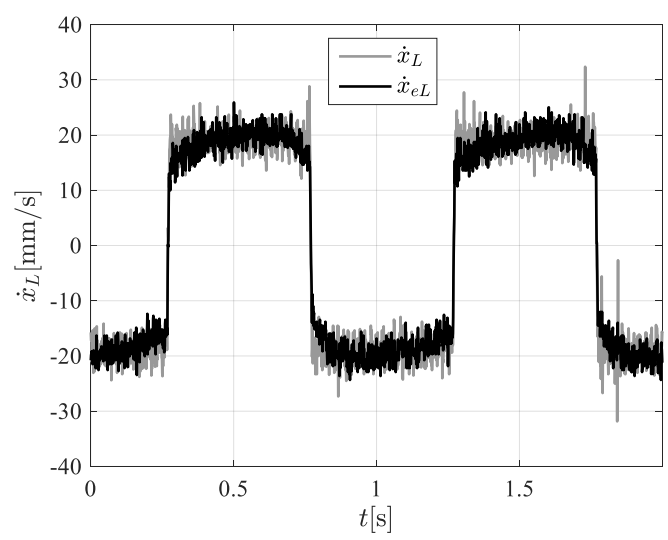

(b) under the load

Fig. 11. Time history of velocity measured and reconstructed from emf; triangle input, $1 \mathrm{~Hz}$

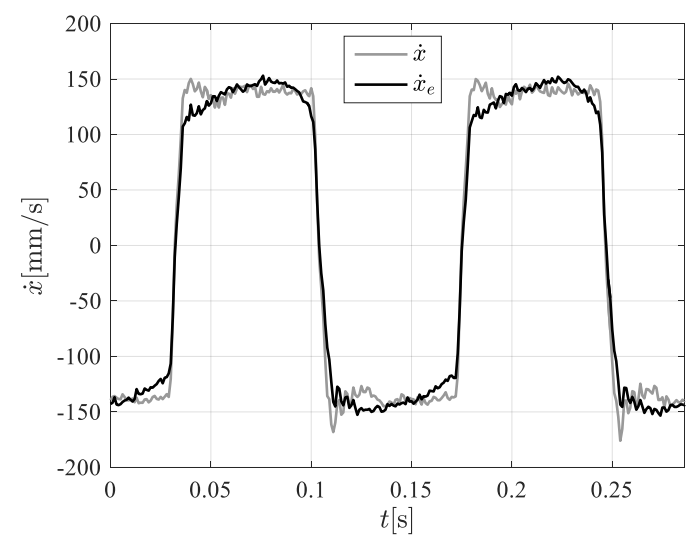

(a) under idle run

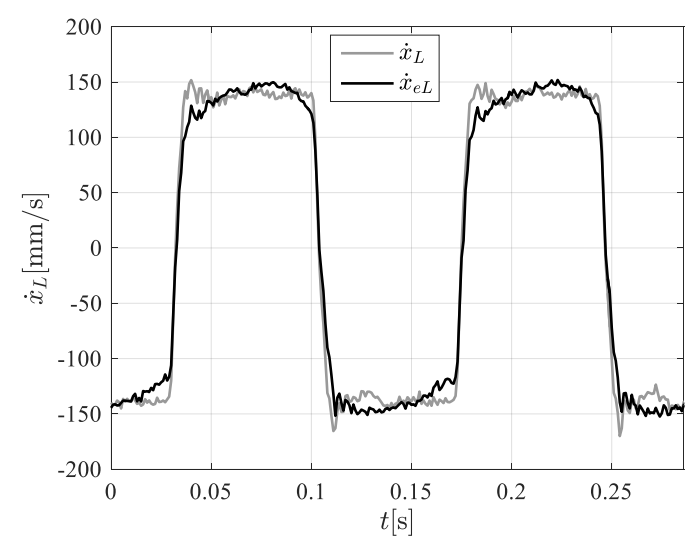

(b) under the load

Fig. 12. Time history of velocity measured and reconstructed from emf; triangle input, $7 \mathrm{~Hz}$

\subsection{Implementation and application of self-sensing function}

The proposed and duly verified self-sensing function has been implemented and tested in a STM32F407 processor embedded system. For the purpose of testing, the clock and peripheral systems of the processor STM32F407 were duly configured and the adequacy of velocity measurement and velocity $\dot{x}$ reconstruction from emf $e$ was tested. Further, testing was done to investigate the temperature correction of parameters $a$ and $b$ in the harvester characteristic and the effectiveness of the sky-hook algorithm. 


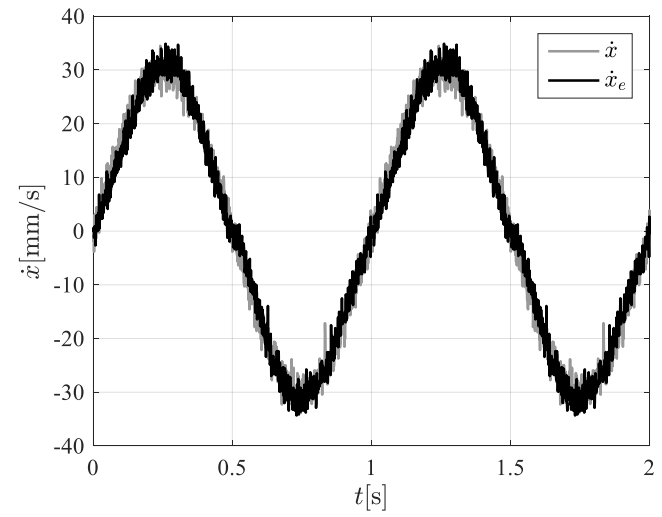

(a) $f=1 \mathrm{~Hz}$

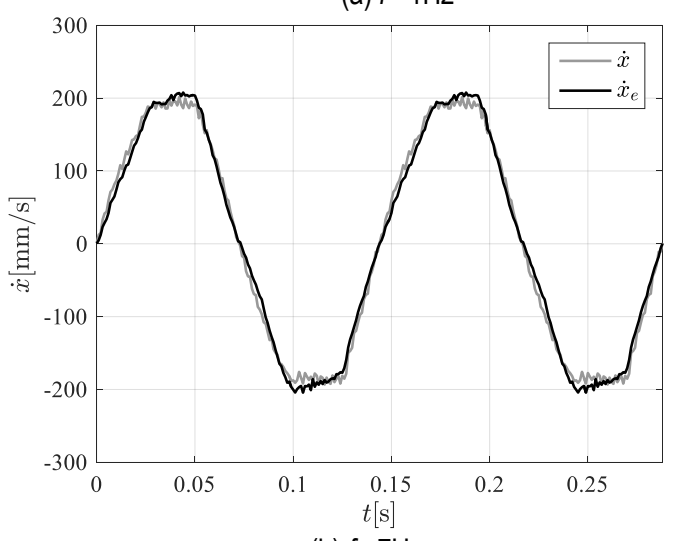

(b) $f=7 \mathrm{~Hz}$

Fig. 13. Time history of velocity measured and reconstructed from emf signal; sine input

Figs. 13a, 13b show time histories of velocity $\dot{x}$ and velocity $\dot{x}_{e}$ derived in accordance with algorithm (1), initiated upon the processing unit for sine wave inputs at frequency 1 and $7 \mathrm{~Hz}$. It appears that the results agree well with those given in Fig. 10, which confirms the effectiveness of channel configuration and the adequacy of the self-sensing function.

To illustrate the correction to the characteristic $\dot{X}_{1}=g\left(E_{1}\right)$, emf generated by the harvester is assumed to be temperaturedependent. This effect is attributable to the reduced magnetisation of ferromagnetic steel with increasing temperature (Kittel, 1996). The proposed correction of the self-sensing function with respect to the measured temperatures involves the modification of values of coefficients $a$ and $b$, according to the identified characteristics $a(T)$ and $b(T)$. Fig. 14 plots time histories of temperature and the values of coefficients $a$ and $b$. Apparently, the value of $a$ tends to increase with increasing temperature, thus compensating for the decrease of the emf generated by the harvester. In the investigated temperature range, the value of coefficient $b$ remains almost unchanged. The temperature-dependence of $a$ and $b$ is illustrated in Figs. 15, 16.

The operating principle of the sky-hook control algorithm (5) running on STM32F407 under the applied sine inputs with frequency $1 \mathrm{~Hz}$ and $7 \mathrm{~Hz}$ is presented in Fig. 17a, 17b. The value of control $u_{s k y}$ is effected through comparing the readouts of instantaneous sprung mass velocity $\dot{x}_{s}$ and reconstructed velocity $\dot{x}_{e}$ values. When the energy in the system is to be dissipated, the value of control voltage $u_{s k y}$ is set equal to $u_{s}$, producing the current in the MR damper coil. At the remaining time instants when the velocity product $\dot{x}_{s} \cdot \dot{x}_{e}<0$, the control value $u_{s k y}=$ 0 , which corresponds to the current level equal to 0 .

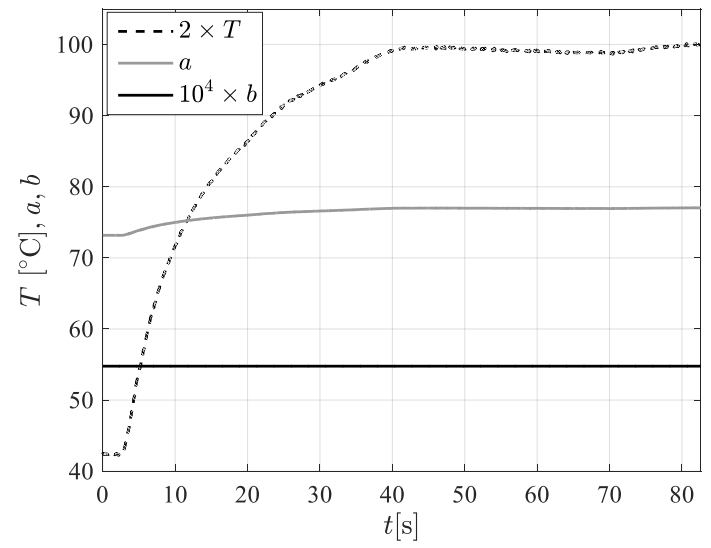

Fig. 14. Time history of temperature, coefficient $a$ and $b$

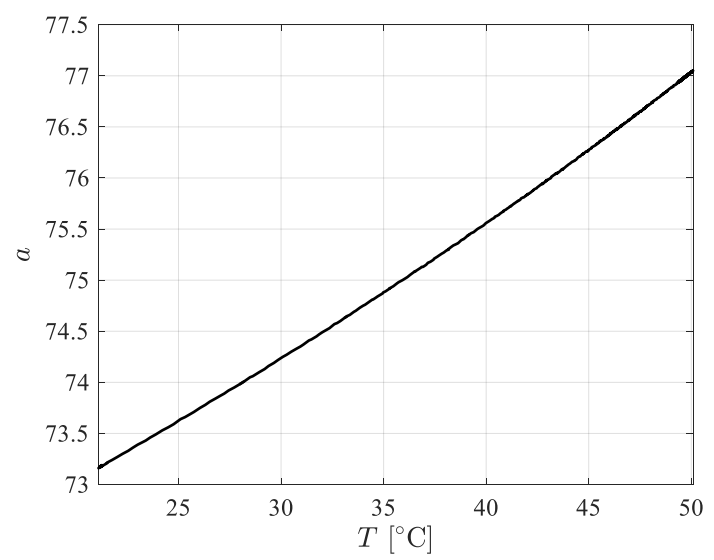

Fig. 15. Coefficient a versus temperature

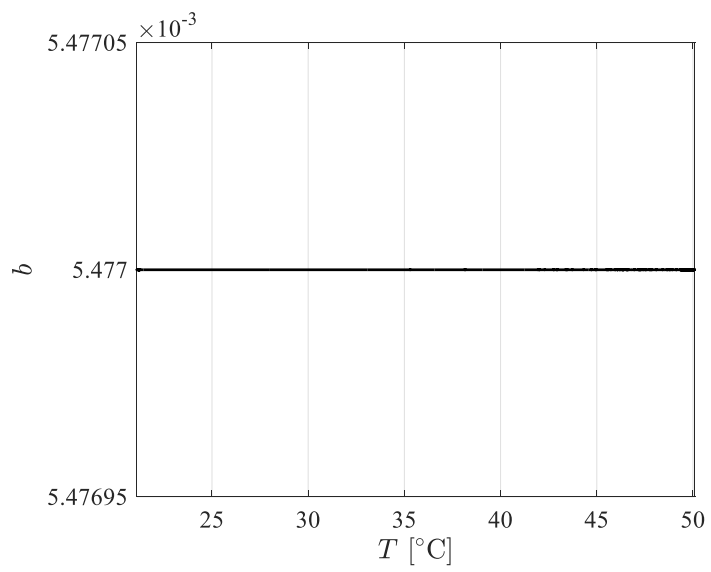

Fig. 16. Coefficient $b$ versus temperature

Fig. 18 shows time histories of logic states for four STM32F407 processor digital outputs used for measuring the times: $T_{A}, T_{C}, T_{S}$ and $T_{0}$. The logic state of the output was pre-set prior to the execution of the analysed code section, and then, reset after the task completion. The beginnings and ends of the measurement procedure: $T_{A}, T_{C}, T_{S}$ and $T_{0}$ are indicated with arrows in Fig. 18. The time histories were registered with a logic analyser at the frequency $50 \mathrm{MSample} / \mathrm{s}$. 


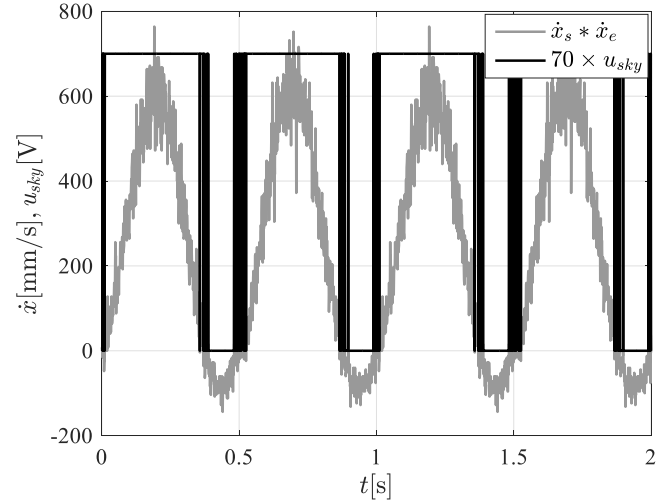

(a) $f=1 \mathrm{~Hz}$

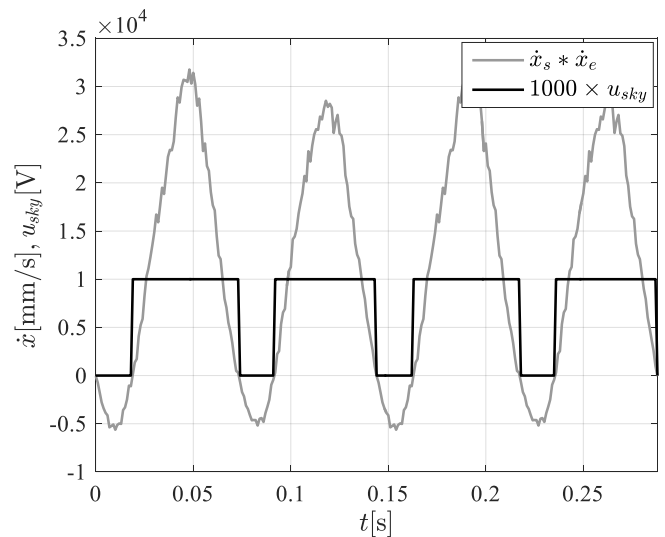

(b) $f=7 \mathrm{~Hz}$

Fig. 17. Calculated velocity product and control; sine input

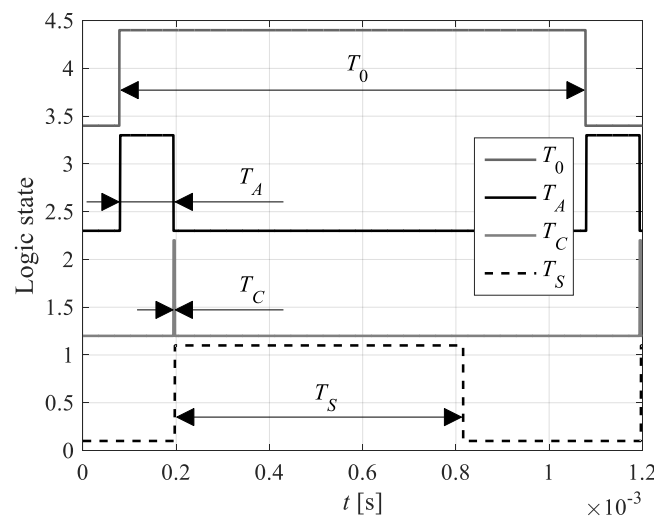

Fig. 18. Measurement of times $T_{0}, T_{A} \mathrm{i} T_{S}$ using logic analyser

A single measurement of $T_{A}, T_{C}, T_{S}$ and $T_{0}$ is insufficient to confirm the time stability involved in particular phases of application code/algorithm execution; that is why, the measurement procedure had to be repeated several time. Results are summarized as histograms in Figs. 19-22. The velocity reconstruction time duration $T_{A}$ is found to be $114.3 \times 10^{-6}$ with jitter $10 \times 10^{-9} \mathrm{~s}$, the control algorithm time duration $T_{C}$ is equal to $2.64 \times 10^{-6} \mathrm{~s}$ with jitter $80 \times 10^{-9} \mathrm{~s}$. The time of data transmission $T_{S}$ from the processing unit to the computer is equal to $620 \times 10^{-6} \mathrm{~s}$ with jitters 2 $\times 10^{-6} \mathrm{~s}$. The sample period $T_{0}$ equals $1 \times 10^{-3} \mathrm{~s}$ with jitter $1 \times 10^{-6}$ s. Actually, the sampling period in $T_{A}, T_{C}, T_{S}$ and $T_{0}$ measurements can be taken as constant because the jitter is attributed mostly to the adopted method of measurements. The sum of control algorithm time duration and of data transmission time is found to be less than $T_{0}$. Actually, the final solution of the embed- ded system will not involve data transmission to a PC, which will vastly reduce the processor time, which should be spent on the implementation of advanced algorithms for correcting the characteristic $\dot{x}=f(e)$ and control of the EHMRD.

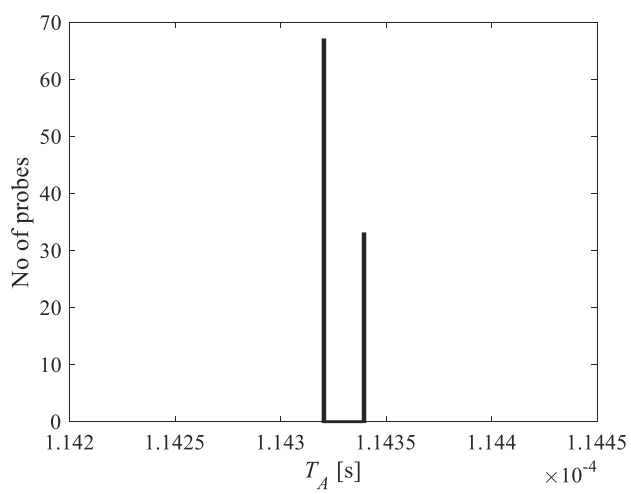

Fig. 19. Histogram of velocity reconstruction time duration $T_{A}$

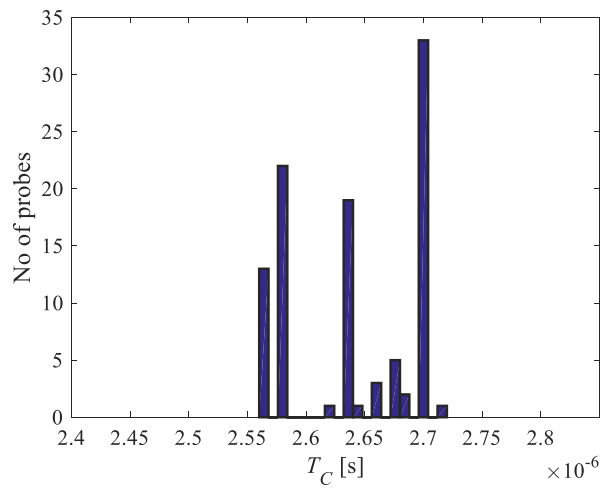

Fig. 20. Histogram of control algorithm time duration $T_{C}$

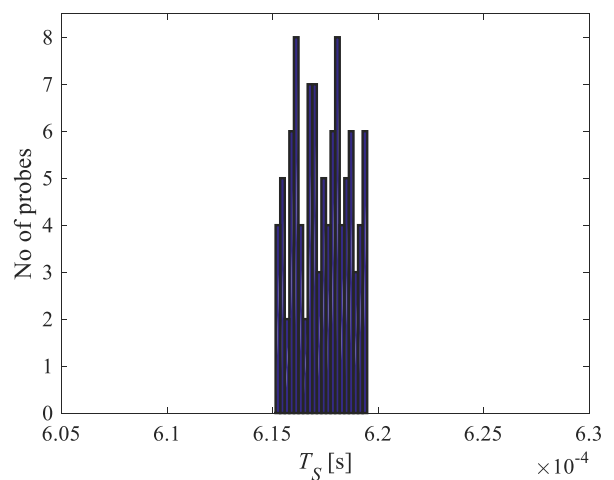

Fig. 21. Histogram of data transmission time $T_{S}$

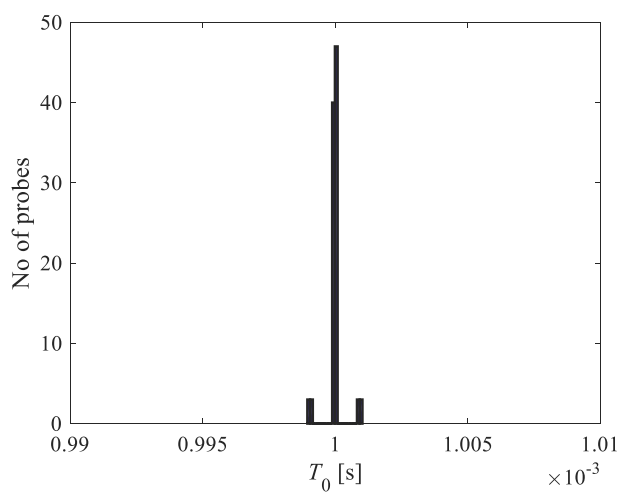

Fig. 22. Histogram of sampling time $T_{0}$ 
Power required to supply the measurement and processing unit determined using a power-supply DP832 (RIGOL, 2016) and a digital multimeter DM3058 (RIGOL, 2015) was found to be 0.4 W (supply voltage: +5 VDC, current: $80 \mathrm{~mA}$ ). The power level was measured for the CPU operated in the run mode at $168 \mathrm{MHz}$, while the peripheral systems were active and supported by the application. The predicted current uptake by the CPU unit obtained in CubeMX taking into account all peripheral systems and GPIO becomes $46 \mathrm{~mA}$ (supply voltage $+3.3 \mathrm{VDC}$, yielding the power ratings of. $0.15 \mathrm{~W}$ ). Results summarized in the work (Sapinski and Rosół 2016) confirm that the EHMRD is capable of generating power sufficient to supply measurement and processing units. Power is generated for Coil $\mathrm{H} 1$ or Coil $\mathrm{H} 2$ at frequencies in excess of $2.5 \mathrm{~Hz}$, with the amplitude $4 \mathrm{~mm}$.

\section{CONCLUSIONS}

The primary objective of the study was to demonstrate that the EHMRD can act effectively as a velocity sensor and is applicable in semi-active vibration control systems. Accordingly, the selfsensing function is derived enabling the velocity EHMRD to be reconstructed from the emf induced in the harvester coil. The proposed function was verified, implemented and tested in the embedded system based on a STM32F407 processor.

The results lead us to the following conclusions:

- As regards the technical aspects, the proposed self-sensing function ensures the satisfactory levels of accuracy in reconstruction of velocity signals from emf measured in the harvester coil.

- Verification of the proposed self-sensing function revealed that a first-order polynomial can be well used in velocity reconstruction from emf readouts.

- The actual values of the self-sensing function parameters obtained in the time domain and in frequency domain allow the velocity reconstruction with similar accuracy levels (the maximum relative difference between the error values equals $3 \%$ ).

- Time instants in which the velocity sign is changed are determined with sufficient accuracy allowing the implementation of switching algorithms,

- Implementation of the self-sensing function in the embedded system showed the results to be identical with those obtained using MATLAB.

- Efficiency of the embedded system is sufficiently high to allow for implementation of advanced strategies for control of EHMRD and for distribution of energy recovered by the harvesting component.

- To improve the accuracy of velocity reconstruction for piston rod inputs in the range $(1,3) \mathrm{Hz}$, it is required that emf signals should be low-pass filtered.

- Implementation of the self-sensing function in the embedded system, including the analogue signal measurement procedure, enables the 10-fold oversampling of emf measurements and operations on medium values.

- Analysis of the sample time period, time duration of velocity reconstruction from emf and time duration of control algorithm confirms the applicability of the embedded system to harvester control at vibration frequencies up to $200 \mathrm{~Hz}$.

In further studies, the proposed self-sensing function used for velocity reconstruction and implemented on an embedded system will be applied in EHMRD-based vibration control system.

\section{REFERENCES}

1. Ahamed R., Ferdaus M.M., Li Y. (2016), Advancement in energy harvesting magneto-rheological fluid damper: A review, KoreaAustralia Rheology Journal, 28(4), 355-379.

2. Ahamed R., Rashid M.M., Ferdaus M.M., Yusuf H.B. (2017), Modelling and performance evaluation of energy harvesting linear magnetorheological (MR) damper, Journal of Low Frequency Noise Vibration and Active Control, 36(2), 177-192.

3. Chen C., Liao W.H. (2012), A self-sensing magnetorheological damper with power generation, Smart Materials and Structures, 21 025014.

4. Chen Z. H., Ni Y.Q., Or S.W. (2015), Characterization and modeling of a self-sensing MR damper under harmonic loading, Smart Structures and Systems, 15, 1103-1120.

5. Choi Y.T., Werely N.M. (2009), Self-powered magnetorhelogical dampers, Journal of Vibration Acoustics, 131, 044501.

6. Frigo M., Johnson S.G. (2005), The Design and Implementation of FFTW3, Proceedings of the IEEE, 93 (2), 216-231. Invited paper, Special Isue on Pro-gram Generation, Optimization, and Platform Adaptation, http://www.fftw.org/fftw-paper-ieee.pdf.

7. Hu G., Lu Y., Sun S., Li W. (2017), Development of a self-sensing magnetorheological damper with magnets in-line coil mechanism, Sensors and Actuators A: Physical, 255, 71-78.

8. Jung H.J., Jang D.D., Lee H.J., Lee I.W., Cho S.W. (2010a), Feasibility Test of Adaptive Passive Control System Using MR Fluid Damper with Electromagnetic Induction Part, Journal Engineering Mechanics, 136(2), 254-259.

9. Jung H.J., Jang D.D., Koo J.H., Cho S.W. (2010b), Experimental Evaluation of a 'Self-Sensing Capability of an Electromagnetic Induction System Designed for MR Dampers, Journal of Intelligent Material Systems and Structures, 21, 837-836.

10. Karnopp D.C., Crosby M.J., Harwood R.A. (1974), Vibration control using semi-active force generator, ASME Journal of Engineering for Industry, 96(2), 619-626.

11. Kittel C. (1996), Introduction to solid state physics, John Wiley \& Sons, Inc., Eighth Edition.

12. Li Z., Zhuo L., Kuang J., Luhrs G. (2013a), Energy-Harvesting Shock Absorber with a Mechanical Motion Rectifier, Smart Materials and Structures, 22, 028008.

13. Li Z., Zhuo L., Luhrs G., Lin L., Qin Y. (2013b), Electromagnetic Energy harvesting shock absorbers: design, modeling and road tests, IEEE Transactions Vehicle Technology, 62, 1065-74.

14. Liao W.H., Chen C. (2010), Self-powered, sensing magnetorheological dampers US Patent Application, 12/896,760.

15. MTS System Corporation (2006), MTS 810 \& 858 Material Testing Systems, Technical Documentation.

16. Ni Y.O., Chen Z., Or S.W. (2015), Experimental Identification of a Self-Sensing Magnetorheological Damper Using Soft Computing, Journal of Engineering Mechanics, 141(7) 04015001.

17. Peng G., Li W., Hu G., Alici G. (2011), Design and simulation of a self- sensing MR damper, 15th International Conference on Mechatronics Technology, 112-117.

18. Polytec Inc. (2005) OFV-505/503 Vibrometer Sensor Head, Technical Data, https://www.polytec.com.

19. RIGOL Technologies Inc. (2016), DP800 Series Programmable Linear DC Power Supply, Technical Data, https://www.rigol.com.

20. RIGOL Technologies Inc. (2015), DM3068 $6 \quad 1 / 2$ digits Digital Multimeter, Technical Data, https://www.rigol.com.

21. Sapiński B. (2008), An experimental electromagnetic induction device for a magnetorheological damper, Journal of Theoretical and Applied Mechanics, 46, 4, 933-947.

22. Sapiński B. (2010), Vibration power generator for a linear MR damper, Smart Materials and Structures, 19, 105012. 
23. Sapiński B., Krupa S. (2013), Efficiency improvement in a vibration power generator for a linear MR damper: numerical study, Smart materials and Structures, 22, 045011.

24. Sapinski B. (2014), Energy harvesting MR linear damper: prototyping and testing, Smart Materials and Structures, 23, 035021.

25. Sapinski B., Rosół M., Węgrzynowski M. (2016), Investigation of an energy harvesting MR damper in a vibration control system, Smart Materials and Structures, 25, 125017.

26. STMicroelectronics (2017), STM32F405/415, STM32F407/417, TM32F427/437 and STM32F429/439 advanced ARM®-based 32-bit MCUs, RM0090 Reference manual, Rev. 15.

27. Wang D.H., Bai X.X., Liao W.H. (2010), An integrated relative displacement self-sensing magnetorheological damper: prototyping and testing, Smart Materials and Structures, 19, 105008.

28. Wang D.H., Bai X.X. (2013), A magnetorheological damper with an integrated self-powered displacement sensor, Smart Materials and Structures, 22, 075001.
29. Xinchun G., Yonghu H., Yi R., Hui L., Jinping Q. (2015), A novel self-powered MR damper: theoretical and experimental analysis, Smart Materials and Structures, 24, 105033.

30. (2017), A novel velocity self-sensing magnetorheological damper: Design, fabricate, and experimental analysis, Journal of Intelligent Material Systems and Structures, 26, 527-540.

31. Zhu S.Y., Shen W.A., Xu Y.L., Lee W.C. (2012), Linear electromagnetic devices for vibration damping and energy harvesting: modeling and testing, Engineering Structures, 34, 198-212.

Acknowledgment: This work is supported by AGH University of Science and Technology under research program No. 16.16.130.942. 